\title{
Monoclonal antibodies against autocrine motility factor suppress gastric cancer
}

\author{
HAHN-SUN JUNG ${ }^{1}$, SU IN LEE ${ }^{2}$, SEUNG-HOON KANG ${ }^{1}$, JIN SANG WANG ${ }^{1}$, EUN HEE YANG ${ }^{1}$, \\ BYUNGWOOK JEON ${ }^{1}$, JAYHYUK MYUNG ${ }^{1}$, JI YOUNG BAEK ${ }^{2}$ and SONG-KYU PARK ${ }^{2,3}$ \\ ${ }^{1}$ Boryung Central Research Institute, Boryung Pharmaceutical Co. Ltd., Ansan-Si, Kyeongki-Do 03127; \\ ${ }^{2}$ College of Pharmacy, Republic of Korea University, Sejong 30019; ${ }^{3}$ Research Driven Hospital, \\ Korea University Guro Hospital, Biomedical Research Center, Seoul 08308, Republic of Korea
}

Received December 4, 2015; Accepted February 17, 2017

DOI: $10.3892 / 01.2017 .6037$

\begin{abstract}
Autocrine motility factor (AMF), which is a secreted form of phosphoglucose isomerase, is mainly secreted by various tumors and has cytokine-like activity. AMF is known to stimulate proliferation, survival and metastasis of cancer cells, and angiogenesis within a tumor. The present study investigated whether inhibition of AMF using targeted-antibodies was able to suppress the growth of cancer. A migration assay using a Boyden chamber was utilized to measure the activity of AMF on the motility of cancer cells. A recombinant human AMF (rhAMF) prepared from $E$. coli transformed with the pET22b-AMF vector increased the motility of MDA-MB-231 and A549 cells, but it did not affect that of NCI-N87 or HepG2 cells, which exhibited the ability to secrete high amounts of their own endogenous AMF into the culture medium. The extent to which the AMF receptor was expressed on cancer cells did not correlate clearly with the cell motility stimulated by rhAMF. In A549-xenografted nude mice treated with sunitinib or cetuximab, a decrease in the plasma AMF concentration was accompanied by a reduction in tumor weight, suggesting an association between the plasma AMF concentration and anticancer activity. A monoclonal antibody $(9 \mathrm{~A}-4 \mathrm{H})$, which revealed a high binding affinity for E. coli-derived rhAMF, significantly suppressed the growth of tumors in Balb/c nude mice transplanted with the human gastric cancer cell line NCI-N87, to the similar extent as trastuzumab, an anticancer antibody. The present
\end{abstract}

Correspondence to: Dr Song-Kyu Park, College of Pharmacy, Republic of Korea University, 2511 Sejong-ro, Sejong 30019, Republic of Korea

E-mail: spark123@korea.ac.kr

Dr Hahn-Sun Jung, Boryung Central Research Institute, Boryung Pharmaceutical Co. Ltd., 1122-3 Shingil-Dong, Danwon-Gu, Ansan-Si, Kyeongki-Do 03127, Republic of Korea

E-mail: cleojung@boryung.co.kr

Key words: autocrine motility factor, monoclonal antibody, NCI-N87, A549, anticancer activity study suggests, for the first time, that an antibody specific to AMF may be a therapeutic agent for gastric cancer.

\section{Introduction}

Phosphoglucose isomerase (PGI), also termed phosphohexose isomerase (PHI), is a glycolytic enzyme that catalyzes the interconversion of glucose-6-phosphate and fructose-6-phosphate in the glycolysis process. A secreted version of PGI/PHI, which has cytokine activity, was originally purified from the conditioned culture medium of human melanoma cells and was termed autocrine motility factor (AMF) owing to its ability to stimulate cell motility (1). Until the respective protein sequences were determined, AMF was termed neuroleukin, a maturation factor mediating differentiation of human myeloid leukemia cells, a myofibril-bound serine proteinase inhibitor, and sperm antigen-36 (2-5). Although the secretion of AMF by normal cells has not been observed, its secretion into culture medium has been identified in various cancer cells. Clinical studies measuring AMF in the serum or urine of patients with lung, gastrointestinal and renal cancer, have suggested that it may be a useful biomarker for certain types of cancer $(6,7)$. In addition to the cell motility stimulating activity, AMF is involved in cellular proliferation (8), cellular survival (9), invasion of malignant cells (10), tumor metastasis (11) and the induction of angiogenesis (12).

AMF binds to and stimulates the AMF receptor (AMFR), which is a $78 \mathrm{kDa}$ glycoprotein (gp78) with seven transmembrane domains (13). When stimulated by AMF, AMFR activates signal transduction pathways that are involved in enhancing cell motility and proliferation and suppressing cell adhesion; these include activation of protein kinase $\mathrm{C}$, the activation and upregulation of Rho-like GTPases and the upregulation of SNAIL $(11,14,15)$. A wide range of clinicopathological studies reviewed by Chiu et al (16), have demonstrated that expression of AMFR was significantly elevated in various kinds of tumor cells compared with adjacent normal tissues.

Studies to date reveal that AMF or AMFR may be a therapeutic drug target, however anticancer drugs targeting these cellular molecules have not yet been developed. Several studies have demonstrated that downregulation of 
endogenous AMF, using small interfering RNA, interfered with the normal functions of cancer cells by inhibiting the EGF-induced invasion of breast cancer cells (17) or by inducing mesenchymal-to-epithelial transition of human fibrosarcoma, osteosarcoma and endometrial cancer cells (18-20), suggesting that inhibiting AMF activity or downregulating AMF expression would be a useful approach to treat cancer. In the present study, monoclonal antibodies specific to the recombinant human AMF (rhAMF) were selected from a naive human scFv phage display library and evaluated for their antitumor activity and effects on the plasma level of endogenous AMF. This is the first report demonstrating that antibodies targeting AMF have antitumor activity in human tumor-xenografted mice, as well as causing decreased concentrations of AMF in the plasma.

\section{Materials and methods}

Cell lines. MRC-5, HEK293, HEK293F, K-562, Huh-7, and HepG2 cells were grown in Dulbecco's modified Eagle's medium (Thermo Fisher Scientific, Inc., Waltham, MA, USA) supplemented with $10 \%$ (vol/vol) heat inactivated fetal bovine serum (Thermo Fisher Scientific, Inc.). NCI-N87, A549, and MDA-MB-231 cells were grown in RPMI-1640 supplemented with $10 \%$ heat inactivated fetal bovine serum. HEK293F cells were grown in Expi293 ${ }^{\mathrm{TM}}$ Expression medium (Gibco; Thermo Fisher Scientific, Inc.). All cell lines, with the exception of Huh-7 and HEK293F, were obtained from the ATCC (Manassas, VA, USA). Huh-7 cells were obtained from The Japanese Collection of Research Bioresources Cell Bank (Osaka, Japan) and HEK293F cells were purchased from Thermo Fisher Scientific, Inc.

Preparation of recombinant human AMF. E. coli. BL21 (DE3; \#RH217; Real Biotech Corporation, Banqiao, Taiwan) on ice for $10 \mathrm{~min}$ were transformed with the pET22b (+) vector (\#69744; Merck KGaA, Darmstadt, Germany) containing AMF cDNA, according to the manufacturer's protocol. The transformed E. coli cells in luria broth (Lennox; Sigma-Aldrich; Merck KGaA) were incubated for $4 \mathrm{~h}$ at $37^{\circ} \mathrm{C}$ with agitation $(250 \mathrm{rpm})$, and induced with $0.1 \mathrm{mM}$ isopropyl $\beta$-D-1-thiogalactopyranoside. HEK293F cells were transfected with pcDNA3.1-AMF vector (Thermo Fisher Scientific, Inc.) containing AMF cDNA, using polyethylenimine (PEI; Polysciences, Inc., Warrington, PA, USA). The transiently transfected cells were grown in Expi293 $3^{\mathrm{TM}}$ Expression medium at $37^{\circ} \mathrm{C}$ for 4 days. The transformed E. coli lysate and the transfected HEK293F conditioned culture medium were applied to HisTrap FF (GE Healthcare Bio-Sciences, Pittsburgh, PA, USA) equilibrated with $50 \mathrm{mM}$ Tris buffer ( $\mathrm{pH}$ 7.4) containing $0.5 \mathrm{M}$ sodium chloride. The column-bound fractions were eluted with $50 \mathrm{mM}$ Tris buffer ( $\mathrm{pH} 7.4$ ) containing $0.5 \mathrm{M}$ sodium chloride and $250 \mathrm{mM}$ imidazole, and then dialyzed with PBS (pH 7.4) three times at $201 \mathrm{x}$ g for $10 \mathrm{~min}$ at $4^{\circ} \mathrm{C}$ using VIVA spin 20 (Sartorius AG, Göttingen, Germany) with $30 \mathrm{kDa}$ molecular weight threshold.

SDS-PAGE analysis. The cell lysate of E. coli cells transformed with the pET22b-AMF vector and rhAMF purified from the transformed $E$. coli cells were separated by electrophoresis on a $10 \%$ SDS Tris-glycine polyacrylamide gel. The protein bands were stained with Coomassie brilliant blue R-250 (Bio-Rad Laboratories, Inc., Hercules, CA, USA).

Western blotting analysis. The E. coli cell lysates and the rhAMF-containing column chromatography fractions were separated by $10 \%$ SDS-PAGE and blotted onto a polyvinylidene difluoride membrane using iBlot apparatus (Invitrogen; Thermo Fisher Scientific, Inc.). The blot was blocked with $5 \%$ skimmed milk in PBS ( $\mathrm{pH} 7.4$ ) for $2 \mathrm{~h}$ at room temperature. The blocked membrane was incubated for $1 \mathrm{~h}$ at room temperature with a mouse monoclonal antibody recognizing the His-tag conjugated to rhAMF (\#70796-3; dilution, 1:1,000; Novagen; Merck KGaA), then with a horseradish peroxidase (HRP)-conjugated anti-mouse immunoglobulin $\mathrm{G}$ (IgG) antibody (KPL, Inc., Gaithersburg, MD, USA) for $1 \mathrm{~h}$ at room temperature. The blot was incubated in the presence of tetramethylbenzidine substrate (KPL, Inc.), the reaction was stopped by adding a stop solution (\#50-85-01; KPL, Inc.) and the signal was visualized using ChemiDoc XRS+ with Image $\mathrm{Lab}^{\mathrm{TM}}$ Software (Bio-Rad Laboratories, Inc.).

Measurement of the enzyme activity of AMF. The enzyme activity of a commercial rhAMF (MyBioSource, San Diego, CA, USA) and the purified rhAMFs were measured using a glucose-6-phosphate isomerase activity assay kit (Abcam, Cambridge, UK), according to the manufacturer's protocol.

ELISA for AMF quantification. AMF secreted in the culture medium and AMF in the plasma of Balb/c nude mice were measured using a glucose-6-phosphate isomerase human ELISA kit (Abcam), according to the manufacturer's protocol.

FACS analysis. Cells were suspended at a density of $1 \times 10^{6}$ cells $/ \mathrm{ml}$ in PBS ( $\mathrm{pH} 7.4$ ) and incubated in the presence of a fluorescein isothiocyanate-conjugated anti-AMF receptor (AMFR) antibody (\#ab76841; dilution, 1:1,000, Abcam) for $1 \mathrm{~h}$ at $4^{\circ} \mathrm{C}$ in the dark. Subsequent to incubation, the cells were washed 3 times with PBS and incubated in the presence of goat-anti-rabbit IgG H\&L (Alexa Fluor 488 ${ }^{\circledR}$; \#ab150077, dilution 1:1,000, Abcam) for $1 \mathrm{~h}$ at 4 in the dark. The cells were washed 2 times with stain buffer (\#554656; BD Biosciences, Franklin Lakes, NJ, USA). Subsequently, the cell surface fluorescence was analyzed by flow cytometry using a FACScaliber (BD Biosciences).

Migration assay. Cancer cell motility was measured by a modification of the Boyden chamber assay, using a CytoSelect 96-well cell migration assay kit (Cell Biolabs Inc., San Diego, SD, USA). The MDA-MB-231, A549, NCI-N87 and HepG2 cells $\left(1 \times 10^{6}\right.$ cells $\left./ \mathrm{ml}\right)$ were incubated in the upper chamber, and $10 \mu \mathrm{g} / \mathrm{ml} \mathrm{E}$. coli-derived rhAMF was added to the lower chamber, which was separated by an $8 \mu \mathrm{m}$ pore membrane. After $24 \mathrm{~h}$ incubation, cells on the upper surface of the membrane were removed by scraping, and the cells on the lower surface were detached using cell detachment solution. The detached cells were lysed and quantified using CyQuant GR fluorescent dye (Cell Biolabs, Inc., San Diego, CA, USA), according to the manufacturer's protocol. 
Screening of human monoclonal anti-AMF antibodies. Human monoclonal antibodies against AMF were selected from a naive human $\mathrm{scFv}$ (single chain fragment variable) phage display library (a total of $\sim 10^{11}$ members, A \& R Therapeutics Co., Daejeon, Korea). A total of 4 rounds of biopanning were performed and four clones of anti-AMF antibodies of $9 \mathrm{~A}-4 \mathrm{H}, 7 \mathrm{~F} 4,8 \mathrm{~F}$ and $4 \mathrm{H}$ were isolated. During each round, AMF-specific phages were selected out of the pool by washing away non-specific binders using PBS-T (PBS containing $0.05 \%$ Tween 20). Following 4 rounds, a mixture of highly specific phage clones was separated from a mono clone phage and selected on the basis of affinity and specificity against AMF using indirect ELISA. Briefly, putative AMF-specific phages were added into the well of 96 -well plates coated with rh-AMF and incubated for $2 \mathrm{~h}$ at room temperature. The wells were washed three times with PBS-T and then anti-M13 HRP antibody (\#ab50370; Abcam) was added and incubated for $1 \mathrm{~h}$ at room temperature. Tetramethylbenzidine substrate was added and the reaction was stopped at $10 \mathrm{~min}$ by adding the $\mathrm{ABTS}^{\circledR}$ Peroxidase stop solution kit (\#50-85-01; KPL, Inc.). The plate was measured at a wavelength of $450 \mathrm{~nm}$. The phage vector from each clone was subjected to sequencing to determine the nucleotide sequence of $\mathrm{VL}$ and $\mathrm{VH}$, and then the nucleotide sequences were analyzed and grouped. The VH and VL gene elements of a selected phage were amplified using polymerase chain reaction and then subcloned into mammalian expression vectors (pYG300H and pYG300L, respectively; A \& R Therapeutics Co.), each of which contained a constant region of heavy chain and light chain of human IgG1 coding sequence for fully human IgG1 expression. Sequencing was performed using ABI PRISM 3730XL Analyzer (Applied Biosystems; Thermo Fisher Scientific, Inc.) to confirm in-frame insertion of each variable region into the expression vectors.

Surface plasmon resonance. The antibody-antigen interaction was examined by surface plasmon resonance spectrometry (SR7500DC; Reichert, Inc., Depew, NY, USA). Prior to antigen immobilization, a Polyethylene Glycol/Carboxyl sensor chip (\#13206061; Reichert, Inc.) was first activated with a mixture of 0.1 M 1-ethyl-3- (3-dimethylamminopropryl) carbodiimide hydrochloride and $0.05 \mathrm{M}$-hydroxysuccinimide at a flow rate of $20 \mu \mathrm{l} / \mathrm{min}$ for $10 \mathrm{~min}$. Following the activation of the chip surface, $50 \mu \mathrm{g} / \mathrm{ml}$ antigen was flowed over the chip surface. The antigen was immobilized to the surface of the chip via free amine coupling to the immobilized succinimide, followed by quenching the remaining activated succinimide ester with $1 \mathrm{M}$ ethanolamine ( $\mathrm{pH}$ 8.5). The chip was equilibrated with PBS-T. For the analysis of antibody association, solutions $(0,12.5,25,50,100,200,400$ and $800 \mathrm{nM})$ of anti-AMF antibodies, 9A-4H, 4H, 7F4 and 8F, in PBS-T was flowed over the sensor chip at a rate of $30 \mu \mathrm{l} / \mathrm{min}$ for $5 \mathrm{~min}$. For the molecular dissociation, washing buffer was flowed over the sensor chip at a rate of $30 \mu \mathrm{l} / \mathrm{min}$ for $5 \mathrm{~min}$. The chip was regenerated with $20 \mathrm{mM} \mathrm{HCl}$. The data was analyzed using the Scrubber 2.0 software (BioLogic Software; Bio-Rad Laboratories, Inc.).

A549 or NCI-N87 xenograft in nude mice. The six-week-old $\mathrm{BALB} / \mathrm{c}$ female mice (weighing $20 \mathrm{~g}$; Charles River Laboratories International, Inc., Yokohama, Japan) were housed under specific pathogen free conditions. Food and
Table I. Enzyme activity of rhAMF.

\begin{tabular}{lcc}
\hline $\begin{array}{l}\text { Commercial } \\
\text { rhAMF }\end{array}$ & $\begin{array}{c}\text { E. coli-derived } \\
\text { rhAMF }\end{array}$ & $\begin{array}{c}\text { HEK293F-derived } \\
\text { rhAMF }\end{array}$ \\
\hline 3.65 & 4.64 & 3.50 \\
\hline
\end{tabular}

Values are $\mathrm{mU} / \mathrm{mg}$. rhAMF, recombinant human autocrine motility factor.

water were sterilized prior to use; the light cycles were $12 \mathrm{~h}$ light/dark and the temperature was maintained between 20 and $22^{\circ} \mathrm{C}$. The animal protocol was reviewed by the Korea University Institutional Animal Care \& Use Committee (Seoul, Korea). A549 or NCI-N87 cells suspended in PBS (pH 7.4) were injected subcutaneously in the right flank of the mice at a concentration of $2 \times 10^{7}$ cells $/ \mathrm{ml}$. The intravenous administration of sunitinib ( 4 or $40 \mathrm{mg} / \mathrm{kg}$, orally administered, once a day for 4 weeks; Sigma-Aldrich; Merck MGaA), cetuximab (4 or $40 \mathrm{mg} / \mathrm{kg}$, intraperitoneal, twice a week for 4 weeks; Namyang Pharm Co., Ltd., Seoul, Korea), trastuzumab (15 mg/kg, intravenously twice a week for 2 weeks; Namyang Pharm Co., Ltd., Seoul, Korea) or human monoclonal anti-AMF antibodies (9A-4H, 7F4, 8F and 4H; $15 \mathrm{mg} / \mathrm{kg}$, intravenously twice a week for 2 weeks) against $E$. coli-derived rhAMF, began when the average volume $\left(\mathrm{V}_{0}\right)$ of the tumors reached $\sim 100 \mathrm{~mm}^{3}$. Tumor volumes $\left(\mathrm{V}_{\mathrm{t}}\right)$ were measured 3 times a week for 3 weeks using a vernier caliper, and then calculated by the formula $0.5 \mathrm{x}$ height $x$ length $x$ width. At the end of the experiment the mice were sacrificed by $\mathrm{CO}_{2}$ asphyxiation.

Statistical analysis. GraphPad Prism (GraphPad Software, Inc., La Jolla, CA, USA) was used for statistical analysis. One-way ANOVA and Dunnett's post hoc t-test were used for multiple comparisons. Spearman correlation analysis was used to compare the correlation between plasma AMF concentration and tumor weight in A549-xenografted mice. $\mathrm{P}<0.05$ was considered to indicate a statistically significant difference.

\section{Results}

Preparation and validation of $r h A M F$. To select the best material for the experiments, rhAMFs from 3 different sources were analyzed and compared. The enzyme activity of rhAMFs purified from the $E$. coli lysate transformed with the pET22b-AMF vector, rhAMFs present in the culture medium of HEK293F cells transiently transfected with the human AMF cDNA in the pcDNA3.1-AMF vector, and commercial rhAMF were 4.64, 3.50 and $3.64 \mathrm{mU} / \mathrm{mg}$ (Table I), respectively. The enzyme activity of rhAMFs prepared from $E$. coli and HEK293F cells were similar to that of the commercial rhAMF, which was included in the assay for comparison. The identity of the final product purified by the column chromatography method was validated by SDS-PAGE and western blot analyses (Fig. 1A and B). The lanes of the gel loaded with the commercial rhAMF, the soluble fraction from the pET22b-AMF-transformed E. coli lysate, and the $250 \mathrm{mM}$ imidazole elution fraction revealed clear bands stained with 
A

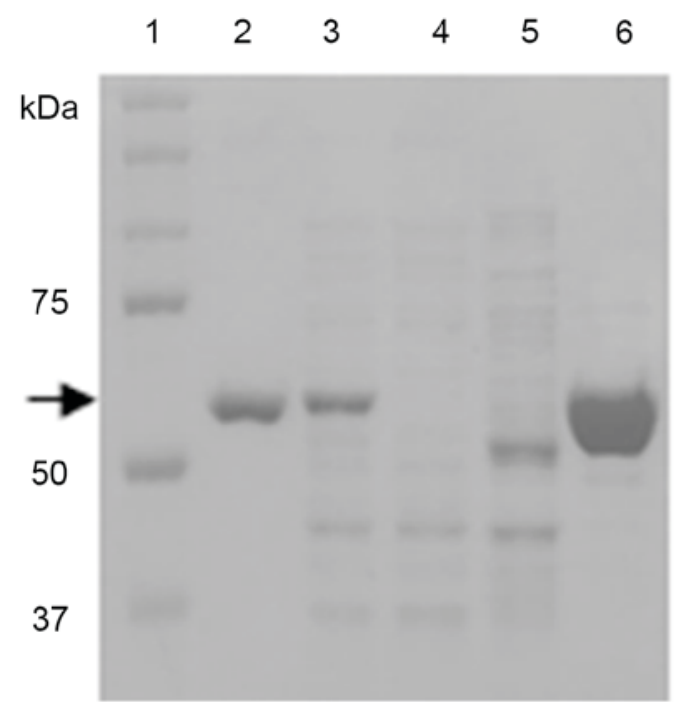

B

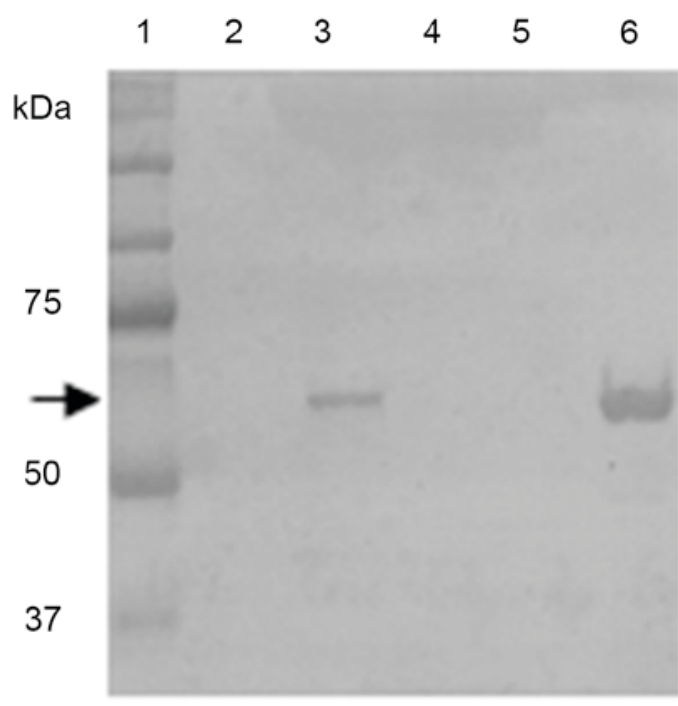

Figure 1. SDS-PAGE and western blot analyses of rhAMF expressed in E. coli. (A) The lysate of E. coli cells expressing rhAMF and the column chromatography fractions were resolved on a $10 \%$ polyacrylamide gel, which was subsequently stained with Coomassie brilliant blue R-250. (B) The gel from (A) was transferred onto a PVDF membrane and analyzed by western blotting. Lane 1, Precision plus protein standard; lane 2, 50 ng commercial rhAMF protein; lane 3, the soluble fraction from the lysate of pET22b-AMF-transformed E. coli; lane 4, flow-through fraction; lane 5, 50 mM imidazole washing fraction; lane $6,250 \mathrm{mM}$ imidazole elution fraction. Arrows indicate the bands for rhAMFs. rhAMF, recombinant human autocrine motility factor.

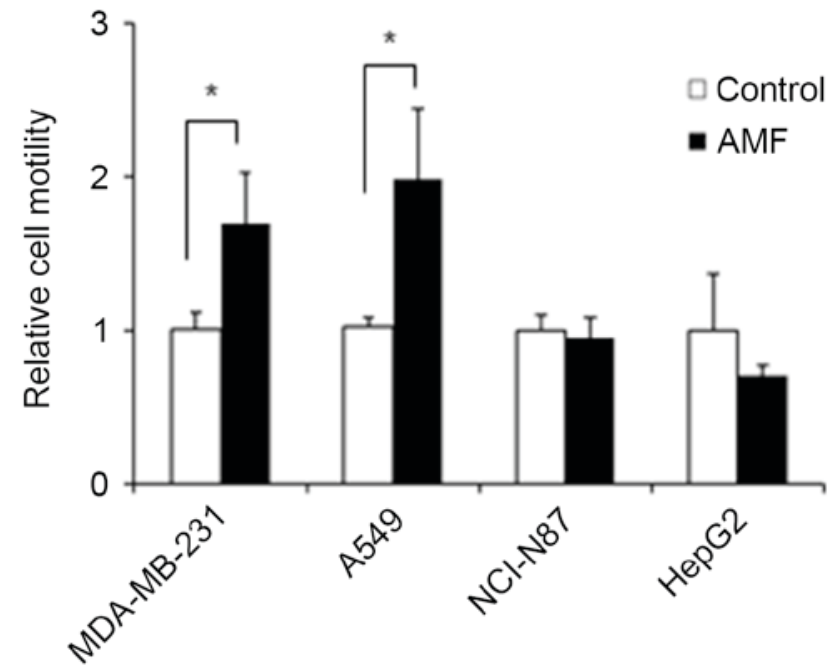

Figure 2. Effect of rhAMF on the migration of cancer cells. The motility of cell lines in the presence and absence of $E$. coli-derived rhAMF was determined using a Boyden chamber assay. The relative motility of cells treated with rhAMF was compared with the motility of untreated cells, whose motility was set at 1 . Results are presented as the mean \pm standard deviation for triplicate determinations. ${ }^{*} \mathrm{P}<0.05$. rhAMF, recombinant human autocrine motility factor.

Coomassie brilliant blue R-250 at $\sim 55 \mathrm{kDa}$, which is the expected molecular weight of rhAMF. The bands were identified to be rhAMF by western blot analysis using a monoclonal antibody against the His-tag. The commercial rhAMF was not detected by the western blot analysis since it does not contain a His-tag (Fig. 1B). Since the enzyme activity of E. coli-derived rhAMF was not lower than that of rhAMFs from other sources, and the preparation of $E$. coli-derived rhAMF is easier than that from HEK293F cells, the following experiment was conducted using $E$. coli-derived rhAMF.
Effects of E. coli-derived rhAMF on the migration of cancer cells. E. coli-derived rhAMF was examined for its chemoattractive activity upon cancer cells using a Boyden chamber. As shown in Fig. 2, the migration of MDA-MB-231 and A549 cells was significantly increased by treatment with rhAMF. The cell motility of MDA-MB-231 and A549 cells as a result of treatment with rhAMF increased by $\sim 1.6$ - and 1.9 -fold, respectively, compared with that of the control cells. However, the migration of NCI-N87 and HepG2 cells was not significantly changed by treatment with rhAMF.

Effect of the expression of the AMF receptor on the secretion of AMF by cancer cells. In order for a signal to be transmitted into cells by extracellular AMF, the presence of AMFR is required. The relative amount of AMFR was measured in the normal human fibroblast MRC-5 and embryonic kidney HEK293 cells, and in the human gastric carcinoma NCI-N87, hepatocellular carcinoma HepG2, lung carcinoma A549, chronic myelogenous leukemia K-562, hepatocellular carcinoma Huh-7, and breast cancer MDA-MB-231 cells using flow cytometry (Fig. 3). The percentage values of cells that express AMFR in HEK293 and MRC-5 cells were 3.57 and $4.93 \%$, respectively. In the case of cancer cells, while the percentage values of cells that express AMFR in K-562, Huh-7, and HepG2 cells were similar to those in the normal cell lines, those in NCI-N87, A549, and MDA-MB-231 cells were $8.71,8.86$, and $11.32 \%$, respectively, which were 1.8 to 3.2 times higher compared with those in the normal cells. In contrast to the expression of AMFR, there were huge differences in the amount of AMF secreted by these cells (Fig. 4). The AMF-specific productivities of NCI-N87 and HepG2 cells were statistically greater $(\mathrm{P}<0.001)$ compared with the other groups of cells. While the AMF-specific productivities of NCI-N87 and HepG2 cells were 0.097 \pm 0.006 and $0.119 \pm 0.011 \mathrm{pg} / \mathrm{cell} / \mathrm{day}$, respectively, those of A549, K-562, Huh-7 and MDA-MB-232 cells were no higher than 

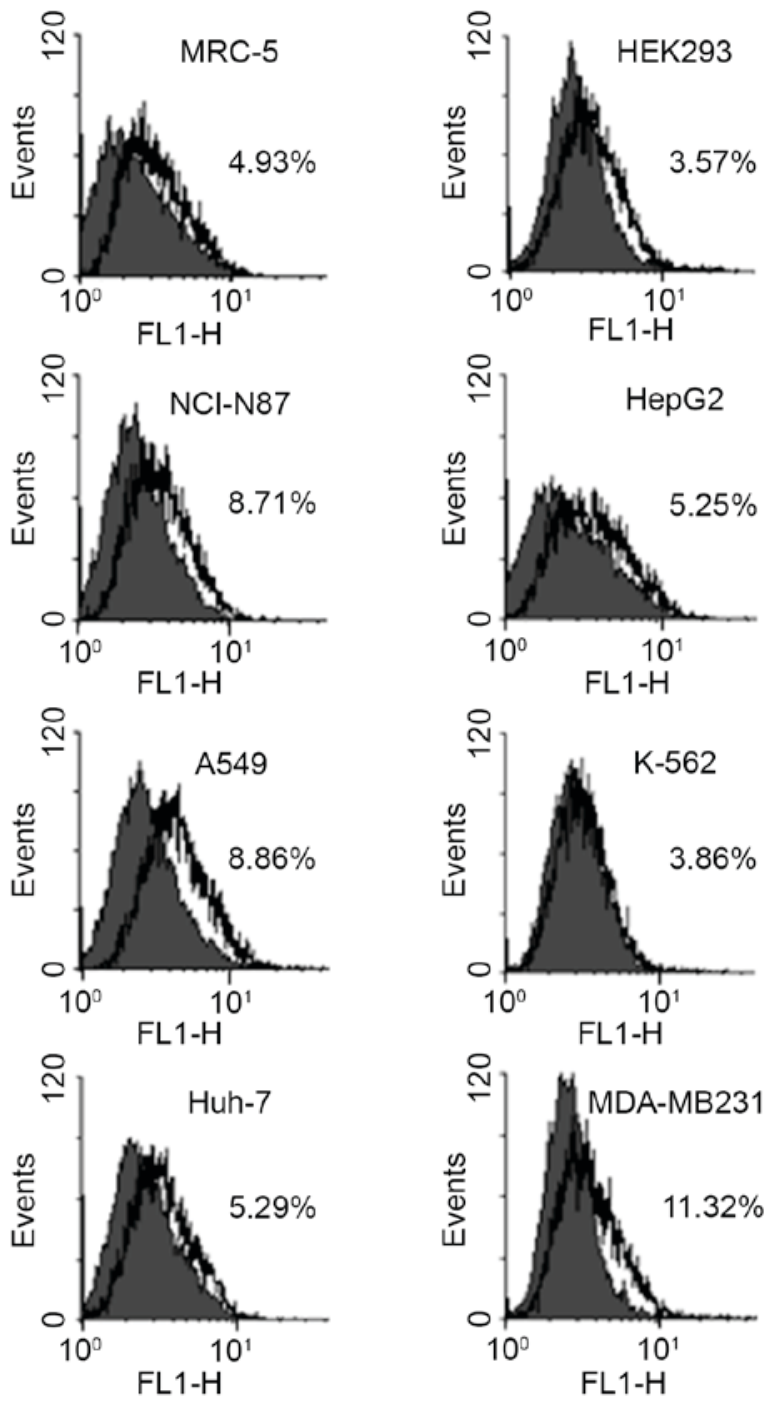

Figure 3. Cell surface expression of the AMFR in various types of cancer cell lines. Cells stained with the fluorescein isothiocyanate-conjugated anti-AMFR monoclonal antibody were applied to flow cytometry analysis. The percentage value from the analysis graph for each cell line denotes the percentage of AMFR expressed in each cell line. The percentage value from each graph denotes the percentage of the total number of cells that express AMFR. AMFR, autocrine motility factor receptor.

$0.037 \pm 0.011 \mathrm{pg} / \mathrm{cell} / \mathrm{day}$. The AMF-specific productivities of the normal cell lines, MRC-5 and HEK293, were 0.012 \pm 0.001 and $0.000 \pm 0.000 \mathrm{pg} / \mathrm{cell} / \mathrm{day}$, respectively. These results were in contrast to those demonstrated in Fig. 2, in that NCI-N87 and HepG2 cells secreted endogenous AMF much more actively than the other cell lines, including MDA-MB-231 and A549 cells, whose motilities were more greatly affected by treatment with rhAMF.

Correlation between plasma AMF concentration and tumor growth. To investigate whether there was a correlation between tumor growth and plasma concentration of AMF, A549-xenografted Balb/c nude mice were treated with sunitinib (21), a small-molecule multi-targeted receptor tyrosine kinase inhibitor, and cetuximab $(22,23)$, a chimeric anti-EGFR monoclonal antibody (Fig. 5). Blood and tumors were collected at the end of the experiments, the plasma concentration of AMF and the tumor weights were measured, and the correlation

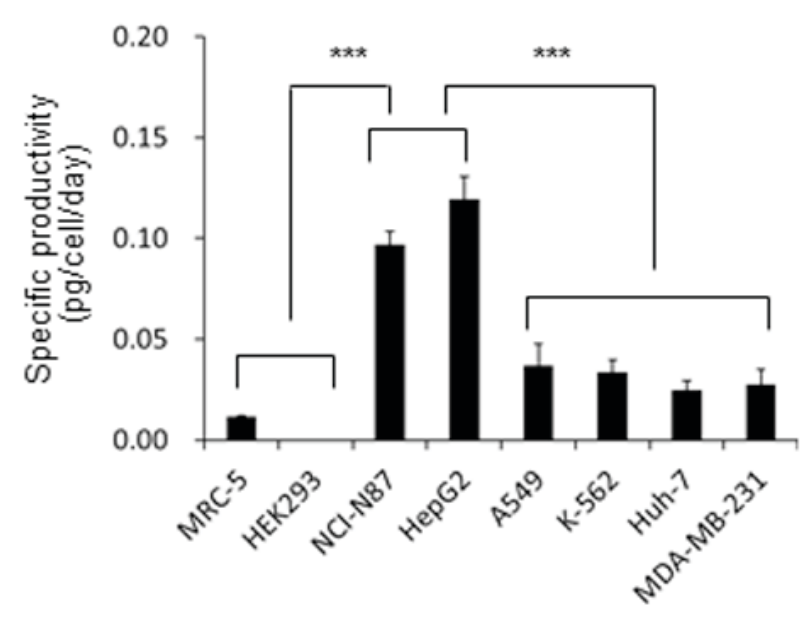

Figure 4. Secretion of AMF by various cell lines. The specific AMF productivities (pg/day/cell) of various types of cells, including normal and cancer cell lines, were determined using ELISA. AMFR, autocrine motility factor receptor. Statistics compared the high AMF secreting and low AMF secreting cells. Results are presented as the mean \pm standard deviation $\left({ }^{* * *} \mathrm{P}<0.001\right)$.

between plasma AMF concentration and tumor weight was analyzed by the Spearman's rank correlation method (Fig. 5). The results reveal that the plasma AMF concentration became lower as the tumor weight decreased upon treatment with sunitinib and cetuximab. As demonstrated with a Spearman's rank coefficient of 0.785 obtained from the analysis of the correlation between the plasma AMF concentration and tumor weight, there was a relatively good correlation between the two factors.

Selection of human monoclonal anti-AMF antibodies. In total, 4 human monoclonal antibodies $(9 \mathrm{~A}-4 \mathrm{H}, 4 \mathrm{H}, 7 \mathrm{~F} 4$ and 8F) specific for AMF were selected from a naive human $\mathrm{scFv}$ phage display library using biopanning. The binding kinetics of the anti-AMF human monoclonal antibodies with E. coli-derived and HEK293F-derived rhAMF were measured using surface plasmon resonance (Table II). The equilibrium dissociation constant $\left(\mathrm{K}_{\mathrm{D}}\right)$ values of these 4 monoclonal antibodies with $E$. coli-derived rhAMF were similar to those with HEK293F-derived rhAMF, however, the $K_{D}$ value of $8 \mathrm{~F}$ with E. coli-derived rhAMF may not be obtained due to a lack of detectable dissociation from the immobilized ligand. Among these 4 antibodies, 9A-4H and 7F4 had somewhat lower affinity for the two rhAMFs than the $8 \mathrm{~F}$ and $4 \mathrm{H}$ antibodies. Since the binding kinetics of these antibodies with rhAMFs derived from two different sources were similar, the following experiments, to investigate the effect of AMF on the migration of cancer cells, were performed with $E$. coli-derived rhAMF, which may be easily prepared compared with the method using the human cell line.

Effects of the monoclonal antibodies on tumor growth and plasmaAMF concentration. Among the 4 monoclonalantibodies (9A-4H, 4H, 7F4, and 8F), only 9A-4H and 7F4 significantly inhibited the growth of tumors in NCI-N87-xenografted mice (Fig. 6A). Monoclonal antibody 9A-4H caused the greatest inhibition among the four monoclonal antibodies, with an inhibition rate slightly higher than that caused by trastuzumab, 
Table II. Surface plasmon resonance binding kinetics of anti-AMF human monoclonal antibodies with rhAMF.

\begin{tabular}{lcccc}
\hline Immobilized ligands & Analytes & $\mathrm{Ka}, \mathrm{M}^{-1} \mathrm{~s}^{-1 \mathrm{a}}$ & $\mathrm{Kd}, \mathrm{s}^{-1 \mathrm{~b}}$ & $\mathrm{~K}_{\mathrm{D}}, \mathrm{nM}^{\mathrm{c}}$ \\
\hline E.coli-derived rhAMF & $9 \mathrm{~A}-4 \mathrm{H}$ & $7.92 \pm 3 \times 10^{3}$ & $1.07 \pm 2 \times 10^{-3}$ & $135 \pm 5$ \\
& $7 \mathrm{~F} 4$ & $4.50 \pm 5 \times 10^{4}$ & $3.71 \pm 3 \times 10^{-3}$ & $82.5 \pm 9$ \\
& $8 \mathrm{~F}$ & $1.23 \pm 2 \times 10^{5}$ & $\mathrm{ND}$ & $\mathrm{ND}$ \\
HEK293F-derived rhAMF & $4 \mathrm{H}$ & $5.67 \pm 5 \times 10^{5}$ & $4.89 \pm 2 \times 10^{-3}$ & $8.64 \pm 7$ \\
& $9 \mathrm{~A}-4 \mathrm{H}$ & $1.22 \pm 3 \times 10^{4}$ & $9.35 \pm 3 \times 10^{-4}$ & $77.0 \pm 2$ \\
& $7 \mathrm{~F} 4$ & $1.06 \pm 2 \times 10^{5}$ & $6.23 \pm 5 \times 10^{-3}$ & $58.9 \pm 8$ \\
& $8 \mathrm{~F}$ & $1.65 \pm 4 \times 10^{5}$ & $1.69 \pm 5 \times 10^{-3}$ & $10.3 \pm 2$ \\
& $4 \mathrm{H}$ & $4.84 \pm 3 \times 10^{5}$ & $8.93 \pm 4 \times 10^{-3}$ & $18.5 \pm 1$ \\
\hline
\end{tabular}

Mean \pm standard error. ${ }^{a}$ Association rate constant. ${ }^{b}$ Dissociation constant. ${ }^{c} \mathrm{Kd} / \mathrm{Ka}$, Equilibrium dissociation constant. ND, no dissociation under assay conditions used; rhAMF, recombinant human autocrine motility factor.

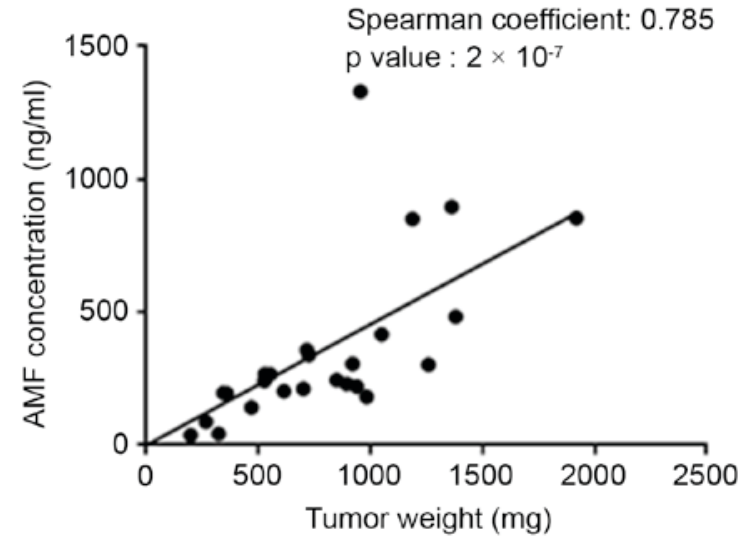

Figure 5. Correlation between the plasma AMF concentration and the tumor weight in A549-xenografted Balb/c nude mice. Blood and tumors were collected from A549-xenografted Balb/c nude mice for the analysis of AMF concentration and the measurement of the tumor weight, 2 weeks subsequent to the final administration of sunitinib ( 4 and $40 \mathrm{mg} / \mathrm{kg}$, orally, once a day for 4 weeks) and cetuximab (4 and $40 \mathrm{mg} / \mathrm{kg}$, intraperitoneal, twice a week for 4 weeks). The statistical analysis for the correlation between the plasma AMF concentration and the tumor weight was analyzed by the Spearman's rank correlation method.

a HER2 inhibitor, which was included as a positive control. The concentration of AMF in plasma collected from the mice at the end of the experiments demonstrated a similar pattern to the inhibition of tumor growth (Fig. 6B). The mean plasma AMF concentration of the Balb/c nude mice treated with $9 \mathrm{~A}-4 \mathrm{H}$ and trastuzumab were significantly decreased compared with that of the control Balb/c nude mice $(\mathrm{P}<0.01)$. Although it was not statistically significant, the mean concentration of AMF in the plasma of the mice treated with 7F4 was decreased compared with the control mice.

\section{Discussion}

The most important characteristics of cancer cells are uncontrolled proliferation and metastatic ability, which allow a tumor to grow indefinitely and transfer to distant organs, respectively. Although there has been certain progress in the improvement of 5-year survival rates of cancer patients due to tremendous effort to elucidate avenues to treat cancers, the majority of cancers, with a few exceptions including thyroid and non-melanoma skin cancer, are still a great threat to humans. Although molecularly-targeted therapeutics, including imatinib (24) and bevacizumab (25), have provided specific evidence that current approaches to cancer treatment are being somewhat successful, newer and improved therapeutics are always requested in the clinic since resistant cancers are being continuously exposed to traditional and the most recent anticancer agents (26). In view of these matters, AMF, which is highly secreted by various cancers, is a valuable anticancer drug target as it is involved in cell proliferation, survival, metastasis and angiogenesis (8-11). The signal stimulated by AMF is transmitted into the cell through AMFR. In previous studies using immunocytochemistry (ICC) staining in tumors obtained from patients, positivity of AMFR expression in the tumor cells was defined when $>10 \%$ of the cells exhibited AMFR staining (27,28). However, in the present study using flow cytometry analysis, only MDA-MB-231 cells, among which $11.32 \%$ were positive, satisfied this criterion. NCI-N87 and A549 cells contained 8.71 and $8.86 \%$ positive cells, which were slightly lower than that of MDA-MB-231 cells, however, the values were increased compared with those of the normal MRC-5 and HEK293 cell lines. The 10\% guideline used to define positivity in ICC staining may not be appropriate for the present study, which used flow cytometry analysis that may be able to analyze the proportion of cells more precisely. It appears that NCI-N87 and A549 cells, as well as MDA-MB-231 cells, are defined as positive in expressing AMFR since the expression level of AMFR in these cell lines was increased compared with that in normal cell lines.

Although it is known that AMF is secreted by various types of cancer, the amount of AMF secreted into the media by cancer cells was greatly variable. Cancer cells originating from digestive organs, including NCI-N87 and HepG2 cells, secreted much more AMF than the other types of cancer cells examined in the present study. However, it is too early to decide if there is an association between the amount of AMF secretion and the organ from which the cancer cells originated. In contrast to the amount of AMF secreted by the cancers, the motility of the cells affected by AMF revealed a different pattern. While the motility of NCI-N87 and HepG2 cells was not increased by treatment with exogenous AMF, the motility of MDA-MB-231 
A

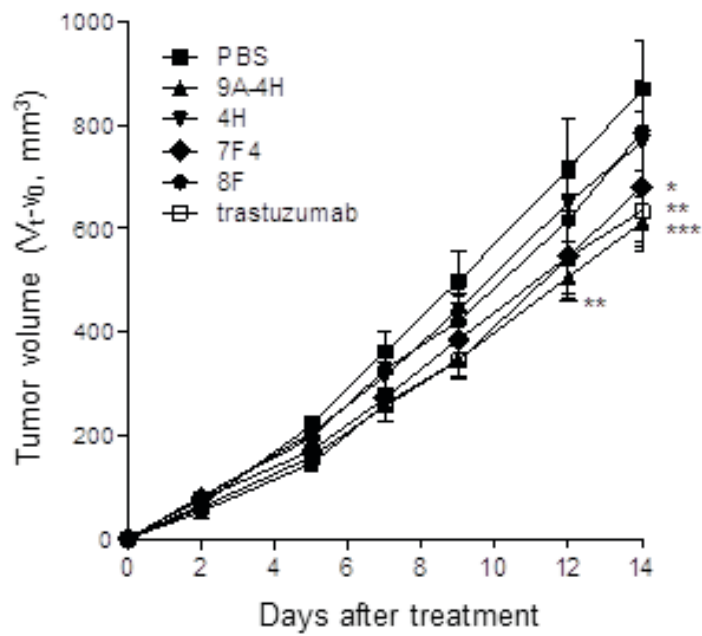

B

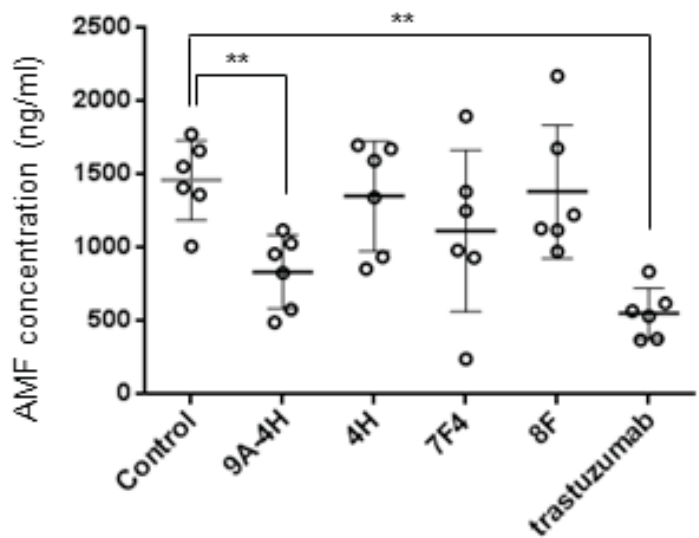

Figure 6. Effects of monoclonal antibodies against rhAMF on tumor growth and plasma AMF concentration in NCI-N87-xenografted Balb/c nude mice. (A) The antibodies and trastuzumab $(15 \mathrm{mg} / \mathrm{kg})$ were administered intravenously twice a week for 2 weeks subsequent to the tumor volume reaching $\sim 100 \mathrm{~mm}^{3}$. Results are presented as the mean \pm standard deviation for hexaplicate determinations $\left({ }^{*} \mathrm{P}<0.05,{ }^{* *} \mathrm{P}<0.01\right.$ and $\left.{ }^{* * * *} \mathrm{P}<0.001\right)$. (B) Plasma AMF concentration was measured at the termination of experiments using ELISA. Results are presented as the mean \pm standard deviation for hexaplicate determinations $\left({ }^{* *} \mathrm{P}<0.01\right)$. rhAMF, recombinant human autocrine motility factor.

and A549 cells was significantly increased. Since there are no studies to date regarding the analysis of the association between the amount of AMF secretion and the amount of AMFR expression in the same cells, it is difficult to say how this happened. One possibility is that AMFR in the cells highly expressing their own AMF, including NCI-N87 and HepG2 cells, may be desensitized. Another possibility is that AMF secreted by cancer cells may produce effects in normal cells near the tumor (29), and subsequently, the normal cells may provide molecules required by the tumor. Thus, in addition to the other previously reported roles of AMF, AMF produced by NCI-N87 and HepG2 cells may serve a role as a paracrine factor, effecting normal cells such as the endothelial cells of blood vessels.

There are studies that AMF has been detected in the urine and plasma of cancer patients $(6,7)$, however, what happens to the level of AMF in the plasma of humans and animal treated with anticancer agents has not been reported. The present study reveals, for the first time, that the plasma concentration of AMF decreased with a reduction in tumor weights in A549-xenografted mice treated with sunitinib or cetuximab. In order to be able to answer the question whether this decrease in plasma AMF concentration contributed to the reduction in tumor weight, further study using AMF inhibitors is necessary.

An antibody specific to AMFR, the 3F3A anti-AMFR IgM monoclonal antibody, has been widely used to identify the presence of this receptor (30), however, it does not have an inhibitory effect on the action of AMF. Antitumor effects of an antibodies specific to AMF have not yet been reported, nevertheless the present study demonstrates that at least two monoclonal antibodies specific to AMF, which were selected from a phage display library, revealed antitumor activity in the NCI-N87-xenografted mouse model, to the same extent as trastuzumab. In addition to tumor growth inhibition, the monoclonal antibody $9 \mathrm{~A}-4 \mathrm{H}$, specific to rhAMF, reduced the plasma concentration of AMF. This result demonstrates that
9A-4H may not only inhibit the action of AMF but may also decrease the secretion of AMF by tumors. Although it is difficult to say how this inhibition of AMF by $9 \mathrm{~A}-4 \mathrm{H}$ is connected to the decrease in the plasma concentration of AMF, there is a possibility that the binding of $9 \mathrm{~A}-4 \mathrm{H}$ interferes with the paracrine or autocrine activity of AMF secreted by the tumor, which is required for further tumor growth. As a result, the growth of tumors decreases, with a subsequent reduction in the amount of AMF secretion.

Trastuzumab is an anticancer agent that was approved for use in patients with HER2-overexpressing breast cancer and HER2-overexpressing metastatic gastric or gastroesophageal junction adenocarcinoma (31). The present study also revealed that trastuzumab decreased the plasma AMF concentration as well as the weight of tumors produced from a gastric cancer cell line. A previous study demonstrated that AMF secretion was inhibited in HER 2 knocked down breast cancer cells, and positively correlated with the overexpression of HER2 (32). The reduction in the plasma concentration of AMF as a result of trastuzumab may have occurred, at least in part, due to its inhibitory effect on HER2 expressed in HER2-positive NCI-N87 cells. Taken together, the present study demonstrates that AMF secretion correlated with tumor weight, and the inhibition of AMF by specific antibodies induced suppression of tumor growth. Although further study is required to obtain a clear understanding regarding the role of AMF in vivo, these results suggest that monoclonal antibody $9 \mathrm{~A}-4 \mathrm{H}$ may be a valuable drug candidate for the treatment of gastric cancer.

\section{Acknowledgements}

This research was supported by the Area-Wide Economic Regional Coalition and Cooperation Program through the Korea Institute for the Advancement of Technology of the Ministry of Trade, Industry and Energy, by the Basic 
Science Research Program through the National Research Foundation of Korea and by the Ministry of Education (grant no. NRF-2014R1A1A2059237).

\section{References}

1. Liotta LA, Mandler R, Murano G, Katz DA, Gordon RK, Chiang PK and Schiffmann E: Tumor cell autocrine motility factor. Proc Natl Acad Sci USA 83: 3302-3306, 1986.

2. Gurney ME, Apatoff BR, Spear GT, Baumel MJ, Antel JP, Bania MB and Reder AT: Neuroleukin: A lymphokine product of lectin-stimulated T cells. Science 234: 574-581, 1986.

3. Xu W, Seiter K, Feldman E, Ahmed T and Chiao JW: The differentiation and maturation mediator for human myeloid leukemia cells shares homology with neuroleukin or phosphoglucose isomerase. Blood 87: 4502-4506, 1996.

4. Cao MJ, Osatomi K, Matsuda R, Ohkubo M, Hara K and Ishihara T: Purification of a novel serine proteinase inhibitor from the skeletal muscle of white croaker (Argyrosomus argentatus). Biochem Biophys Res Commun 272: 485-489, 2000.

5. Yakirevich E and Naot Y: Cloning of a glucose phosphate isomerase/neuroleukin-like sperm antigen involved in sperm agglutination. Biol Reprod 62: 1016-1023, 2000.

6. Gomm SA, Keevil BG, Thatcher N, Hasleton PS and Swindell RS The value of tumour markers in lung cancer. Br J Cancer 58: 797-804, 1988.

7. Baumann M, Kappl A, Lang T, Brand K, Siegfried W and Paterok E: The diagnostic validity of the serum tumor marker phosphohexose isomerase (PHI) in patients with gastrointestinal, kidney, and breast cancer. Cancer Invest 8: 351-356, 1990.

8. Tsutsumi S, Yanagawa T, Shimura T, Fukumori T, Hogan V, Kuwano H and Raz A: Regulation of cell proliferation by autocrine motility factor/phosphoglucose isomerase signaling. J Biol Chem 278: 32165-32172, 2003

9. Tsutsumi S, Hogan V, Nabi IR and Raz A: Overexpression of the autocrine motility factor/phosphoglucose isomerase induces transformation and survival of NIH-3T3 fibroblasts. Cancer Res 63: 242-249, 2003.

10. Haga A, Funasaka T, Deyashiki Y and Raz A: Autocrine motility factor stimulates the invasiveness of malignant cells as well as up-regulation of matrix metalloproteinase-3 expression via a MAPK pathway. FEBS Lett 582: 1877-1882, 2008.

11. Tsutsumi S, Yanagawa T, Shimura T, Kuwano $\mathrm{H}$ and Raz A: Autocrine motility factor signaling enhances pancreatic cancer metastasis. Clin Cancer Res 10: 7775-7784, 2004.

12. Funasaka T, Haga A, Raz A and Nagase H: Tumor autocrine motility factor is an angiogenic factor that stimulates endothelial cell motility. Biochem Biophys Res Commun 285: 118-128, 2001.

13. Silletti S, Watanabe H, Hogan V, Nabi IR and Raz A: Purification of B16-F1 melanoma autocrine motility factor and its receptor. Cancer Res 51: 3507-3511, 1991.

14. Kanbe K, Chigira M and Watanabe $\mathrm{H}$ : Effects of protein kinase inhibitors on the cell motility stimulated by autocrine motility factor. Biochim Biophys Acta 1222: 395-399, 1994.

15. Tsutsumi S, Gupta SK, Hogan V, Collard JG and Raz A: Activation of small GTPase Rho is required for autocrine motility factor signaling. Cancer Res 62: 4484-4490, 2002.
16. Chiu CG, St-Pierre P, Nabi IR and Wiseman SM: Autocrine motility factor receptor: A clinical review. Expert Rev Anticancer Ther 8: 207-217, 2008.

17. Kho DH, Zhang T, Balan V, Wang Y, Ha SW, Xie Y and Raz A Autocrine motility factor modulates EGF-mediated invasion signaling. Cancer Res 74: 2229-2237, 2014.

18. Funasaka T, Hu H, Yanagawa T, Hogan V and Raz A: Down-regulation of phosphoglucose isomerase/autocrine motility factor results in mesenchymal-to-epithelial transition of human lung fibrosarcoma cells. Cancer Res 67: 4236-4243, 2007.

19. Niinaka Y, Harada K, Fujimuro M, Oda M, Haga A, Hosoki M, Uzawa N, Arai N, Yamaguchi S, Yamashiro M and Raz A: Silencing of autocrine motility factor induces mesenchymal-to-epithelial transition and suppression of osteosarcoma pulmonary metastasis. Cancer Res 70: 9483-9493, 2010

20. Li Y, Che Q, Bian Y, Zhou Q, Jiang F, Tong H, Ke J, Wang K and Wan XP: Autocrine motility factor promotes epithelial-mesenchymal transition in endometrial cancer via MAPK signaling pathway. Int J Oncol 47: 1017-1024, 2015.

21. Papaetis GS and Syrigos KN: Sunitinib: A multitargeted receptor tyrosine kinase inhibitor in the era of molecular cancer therapies. BioDrugs 23: 377-389, 2009.

22. Ciardiello F, De Vita F, Orditura M, Comunale D and Galizia G: Cetuximab in the treatment of colorectal cancer. Future Oncol 1 : 173-181, 2005.

23. Hitt R, Martin P and Hidalgo M: Cetuximab in squamous cell carcinoma of the head and neck. Future Oncol 2: 449-457, 2006.

24. Iqbal $\mathrm{N}$ and Iqbal N: Imatinib: A breakthrough of targeted therapy in cancer. Chemother Res Pract 2014: 357027, 2014.

25. Braghiroli MI, Sabbaga J and Hoff PM: Bevacizumab: Overview of the literature. Expert Rev Anticancer Ther 12: 567-580, 2012.

26. Holohan C, Van Schaeybroeck S, Longley DB and Johnston PG: Cancer drug resistance: An evolving paradigm. Nat Rev Cancer 13: 714-726, 2013

27. Takanami I, Takeuchi K, Watanabe H, Yanagawa T, Takagishi K and Raz A: Significance of autocrine motility factor receptor gene expression as a prognostic factor in non-small-cell lung cancer. Int J Cancer 95: 384-387, 2001.

28. Ohta Y, Tanaka Y, Hara T, Oda M, Watanabe S, Shimizu J and Watanabe Y: Clinicopathological and biological assessment of lung cancers with pleural dissemination. Ann Thorac Surg 69: 1025-1029, 2000.

29. Funasaka T and Raz A: The role of autocrine motility factor in tumor and tumor microenvironment. Cancer Metastasis Rev 26: 725-735, 2007

30. Nabi IR, Watanabe H and Raz A: Identification of B16-F1 melanoma autocrine motility-like factor receptor. Cancer research 50 : 409-414, 1990

31. Bang YJ, Van Cutsem E, Feyereislova A, Chung HC, Shen L, Sawaki A, Lordick F, Ohtsu A, Omuro Y, Satoh T, et al: Trastuzumab in combination with chemotherapy versus chemotherapy alone for treatment of HER2-positive advanced gastric or gastro-oesophageal junction cancer (ToGA): A phase 3 , open-label, randomised controlled trial. Lancet 376: 687-697, 2010.

32. Kho DH, Nangia-Makker P, Balan V, Hogan V, Tait L, Wang Y and Raz A: Autocrine motility factor promotes HER2 cleavage and signaling in breast cancer cells. Cancer Res 73: 1411-1419, 2013. 